\title{
Gradhiva
}

Revue d'anthropologie et d'histoire des arts

\section{Laurent Legrain, Chanter, s'attacher et transmettre chez les Darhad de Mongolie}

Paris, Centre d'études mongoles et sibériennes - École pratique des hautes études, coll. « Nord-Asie 4 », 2014

\section{Grégory Delaplace}

\section{(2) OpenEdition}

\section{Journals}

Édition électronique

URL : http://journals.openedition.org/gradhiva/3215

DOI : 10.4000/gradhiva.3215

ISSN : 1760-849X

Éditeur

Musée du quai Branly Jacques Chirac

Édition imprimée

Date de publication : 25 mai 2016

Pagination : 236-237

ISBN : 978-2-35744-093-7

ISSN : 0764-8928

Référence électronique

Grégory Delaplace, "Laurent Legrain, Chanter, s'attacher et transmettre chez les Darhad de Mongolie », Gradhiva [En ligne], 23 | 2016, mis en ligne le 25 mai 2016, consulté le 24 septembre 2020. URL : http:// journals.openedition.org/gradhiva/3215; DOI : https://doi.org/10.4000/gradhiva.3215

Ce document a été généré automatiquement le 24 septembre 2020.

(C) musée du quai Branly 


\section{Laurent Legrain, Chanter, s'attacher et transmettre chez les Darhad de Mongolie}

Paris, Centre d'études mongoles et sibériennes - École pratique des hautes études, coll. « Nord-Asie 4 », 2014

Grégory Delaplace

\section{RÉFÉRENCE}

Laurent Legrain, Chanter, s'attacher et transmettre chez les Darhad de Mongolie. Paris, Centre d'études mongoles et sibériennes - École pratique des hautes études, coll. « Nord-Asie 4 », 2014, 394 p. + CD audio. 

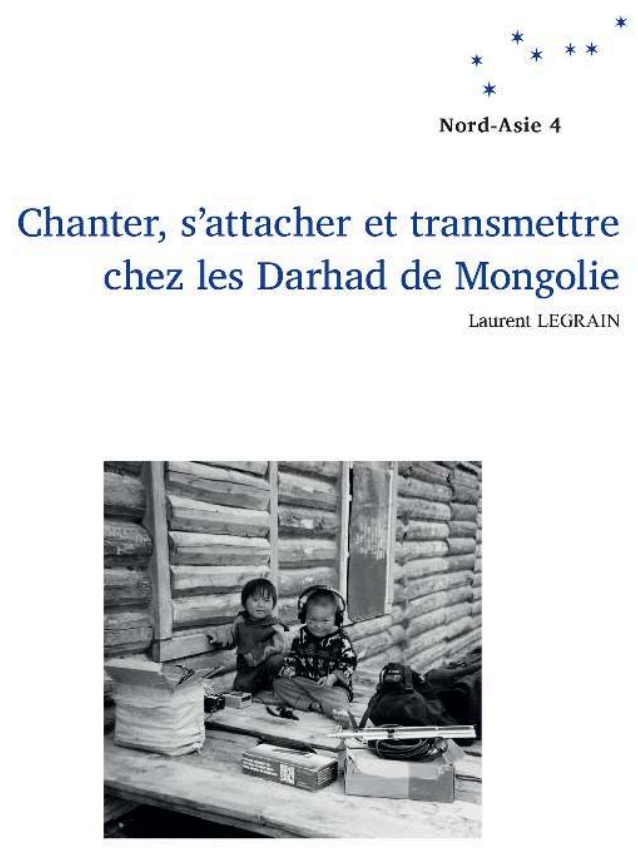

Centre d'Études Mongoles \& Siberiennes - École Pratique des Hautes t́tudes

$1 \quad$ Les Darhad aiment chanter et écouter leurs chants.

2 Le point de départ du livre de Laurent Legrain est si simple qu'il pourrait sembler trivial. Après tout, n'est-ce pas le cas, à des degrés divers, de toutes les sociétés humaines ? En quoi les Darhad, ce peuple de pasteurs nomades vivant à l'orée de la taïga et aux confins de la steppe, dans le nord de la Mongolie, seraient-ils particuliers à cet égard? Il est vrai que le répertoire musical darhad a été célébré depuis les années 1960 par les ethnomusicologues et les folkloristes mongols pour sa richesse et ses formes supposément absentes dans le reste du pays (p. 51). Ainsi, les Darhad se distingueraient des autres groupes ethniques de Mongolie par leurs " chants alternés » (harilcaa duu), et plus encore par leurs "chants moqueurs» (šog duu), ces satires improvisées aux dépens des lamas libidineux, des aristocrates méprisants et autres figures d'autorité, dans lesquelles le régime socialiste s'est plu à lire une forme populaire de critique sociale (p. 57 sq.). D'une manière générale, les Darhad sont célébrés pour la qualité de leurs chants «courts » et «longs » (les deux composantes principales du répertoire national); ils revendiquent eux-mêmes avec force la spécificité de leur style musical et des accents - linguistiques et mélodiques - qui traduisent immanquablement à leur oreille les différents terroirs (nutag) de leurs interprètes et auteurs (p. 51 sq., p. 73 sq.).

À cette spécificité du répertoire chanté darhad, Legrain n'a trouvé, malgré ses efforts et l'insistance des musiciens eux-mêmes, aucun fondement musicologique : «rien ne me permettait de tracer une frontière nette entre le répertoire darhad et celui d'autres minorités [...]. Rien ne me permettait non plus de séparer ce répertoire local de la musique mongole en général» (p. 361). Cette spécificité darhad, au grand dam de l'ethnographe et du folkloriste, est donc largement construite.

4 Indéniablement, pourtant, les Darhad sont attachés à leurs chants. Indéniablement aussi, leur attachement à la spécificité supposée de ce répertoire donne à celui-ci 
consistance et texture. Dès lors, le projet ethnomusicologique se trouve confronté à une alternative, entre les termes de laquelle ce livre décide heureusement de ne pas choisir. En effet, plutôt que de dire que les Darhad se trompent dans leur attachement (en entreprenant de le « déconstruire »), et plutôt que de prendre pour acquise l'existence $\mathrm{du}$ répertoire auquel ils sont attachés (en se contentant de le « documenter »), il prend le parti de déplier, pli après pli, les formes que prend cet attachement et de suivre, médiation après médiation, les relations qui le constituent. Ce que l'on avait pris l'habitude de décrire comme la «musique mongole» s'en trouve radicalement transfiguré. À mesure que l'auteur la réinscrit, chapitre après chapitre, dans une économie générale des sons, sa description s'ouvre sur une étude plus large de l'« épistémologie acoustique » (suivant l'expression de Steven Feld citée p. 362) selon laquelle s'organisent les contacts entre les humains et les sonorités du monde qui éveillent leur attention.

5 L'attachement des Darhad pour le chant est envisagé à partir de trois questionnements, déclinés l'un après l'autre dans l'ouvrage : comment il s'est constitué historiquement, dans quel environnement sonore il s'inscrit, et enfin comment il est transmis.

6 L'auteur commence par examiner dans le détail les dispositifs par lesquels l'amour des Darhad pour leurs chants s'est progressivement tissé - autrement dit, comment les Darhad en sont venus à s'attacher à un répertoire qu'ils considèrent comme ethniquement spécifique (chap. II et III). Il ne suffit pas pour cela de montrer que ces deux réalités - les «Darhad » en tant que groupe ethnique, et le "répertoire darhad» en tant que tradition propre - sont nées au même moment. Il faut aussi comprendre par quelles médiations elles se sont mutuellement constituées depuis les années 1920 : ainsi des institutions comme le «club» (p. 140 sq.), lieu de rassemblement et de performance à partir duquel chaque terroir (nutag) entend apporter sa contribution esthétique à l'édification nationale, ou des pratiques comme la collecte de mélodies et de textes par les responsables des institutions culturelles au moyen de magnétophones et de carnets (p. 150 sq., p. 162 sq.) contribuent à affirmer la réalité d'une musique littéralement populaire - une musique qui émerge de la population même et qui lui est directement destinée. Cette « symbiose entre le chant et le peuple » (p. 169) initiée par les injonctions esthétiques et idéologiques du réalisme socialiste - mais qui finit par en dépasser largement les limites - et produite par les institutions qui en ont émané a participé à instaurer le type d'attachement particulier qui caractérise aujourd'hui le rapport des Darhad à leurs chants, fondé sur l'attention à des différences imperceptibles pour les autres. L'attachement des Darhad en particulier et des Mongols en général pour leurs productions musicales prend la forme de ce que l'auteur appelle, reprenant l'expression de Milan Kundera dans son roman La Plaisanterie, un «tunnel sous l'histoire» (p. 120 sq.) : à l'instar de Jaroslav, l'un des personnages du livre que Legrain érige en idéal-type, ils font tenir l'essence de leur identité culturelle dans les fines spécificités d'une musique qu'il est dès lors indispensable de préserver et de transmettre.

7 Le lien fondamental tracé par l'auteur entre attachement et attention, inspiré des travaux d'Antoine Hennion et véritable fil rouge de l'ouvrage, trouve un nouveau développement dans le deuxième questionnement, qui constitue le cœur de sa proposition théorique : la notion de « continuum sonore» (chap. V). Celle-ci repose sur un fait linguistique apparemment anodin: le terme mongol que l'on traduit habituellement par «chant», duu (prononcez «doux» en étirant un peu le [ou]) 
s'applique aussi à un ensemble de sons, à la voix humaine en premier lieu, mais à d'autres encore dont certains n'entretiennent pas de rapport évident avec le chant ou même les uns avec les autres. Ainsi le roulement des eaux d'une rivière, le sifflement du vent tout comme le rugissement lointain d'un moteur de voiture ou d'un d'hélicoptère sont susceptibles d'être qualifiés de duu. Autrement dit, en mongol, le chant ne s'oppose pas aux autres sons : en tant que son, il s'oppose au «bruit» (šuugian) - la cacophonie des voix d'enfants qui se chamaillent ou le vrombissement devenu assourdissant de l'hélicoptère qui s'apprête à atterrir. Le terme duu, au-delà du chant et de la voix, désigne en fait les sons qui éveillent l'attention - le «bruit » étant par opposition ce qui la parasite (p. 234 sq.).

8 À partir de ce constat, Legrain entreprend de tracer les contours de ce continuum en examinant quelques-uns des sons qui composent l'écosystème acoustique des Darhad. Le vent et les rivières reçoivent une attention particulière tant les différents sons qu'ils produisent sont liés à un «champ de conjectures" (notion que l'auteur emprunte à Caroline Humphrey) sur les états du monde qu'ils permettent de déceler et d'anticiper (p. $247 \mathrm{sq}$.). La voix humaine constitue néanmoins le point d'ancrage principal du continuum sonore (p. 239-247). Legrain décrit la manière dont celle-ci se forme à l'épreuve des sons de l'écosystème d'un terroir particulier: par le chant dont les musiciens éprouvent la force face au torrent de la rivière de leur « pays natal » (törsön nutag), par les huchements et les cris qui rassemblent le bétail et tiennent en respect les loups, ou par les complaintes qui émeuvent les femelles pour les inciter à allaiter le petit qu'elles avaient délaissé (p. 266 sq.). Ainsi, ce n'est pas seulement à leurs chants que les Darhad sont attachés, mais au duu - au continuum des sons vis-à-vis desquels ils cultivent une attention, ou par lesquels ils éveillent l'attention des non-humains qui les entourent, et qui composent au quotidien l'écosystème acoustique dans lequel ils vivent.

9 Le troisième et dernier problème qu'examine l'ouvrage est celui de la transmission: comment se transmet cette attention portée à certains sons et cultivée dans la pratique du chant? Comment les enfants sont-ils conduits à trouver la place de leur voix parmi les autres sons du monde? Outre les « fêtes » (nair), qui sont le lieu par excellence de l'apprentissage et de la circulation des chants (chap. IV), l'auteur détaille les différents « dispositifs» (p. 298) par lesquels les enfants sont encouragés à se positionner dans le continuum sonore (chap. VI). Deux d'entre eux en particulier nous interpellent: les cours de chant dispensés à l'école d'une part, et le gardiennage du troupeau d'autre part, les jeunes bergers étant dès 6 ans envoyés seuls faire paître les moutons. Ce qui rend ces situations comparables, ce n'est pas vraiment que les enfants chantent dans les deux cas, ou pas seulement. Ces deux dispositifs, plus profondément, fonctionnent comme des «niches de développement» (p. 307) au sein desquelles les enfants sont incités par divers « acte[s] de désignation » (p. 295) à porter leur attention sur certains effets possibles de leur voix dans le monde - et inversement, pourrait-on ajouter, sur les signes que constituent les sons de leur environnement. Ainsi, le berger est rendu attentif aux effets de sa voix sur le bétail et les loups, comme l'écolier à ceux que son chant produira sur les émotions de ses parents à son retour chez lui.

Concluons sur cette idée poétique exprimée par les Darhad et développée dans le premier chapitre, selon laquelle le "pays natal» a la texture d'un vêtement souvent porté, dans lequel il est si doux de se glisser. Or, nous dit l'auteur, le pays natal étant composé de la conjonction d'une terre et d'un réseau de personnes qui y sont en fait 
rarement rassemblées, il en manque toujours une part. Le rapport des Darhad à leur terroir, dès lors, est inévitablement investi d'une nostalgie que les chants déclinent sous toutes ses formes et à longueur de strophes. Comme si, nous dit l'auteur, le « doux vêtement » qu'était le pays natal n'était jamais « qu'à moitié enfilé » (p. 47) - comme si l'attachement continuel et profond des Darhad à leurs chants devait pallier «l'incomplétude structurelle» (p. 48) de cette relation.

11 Le livre de Legrain est aussi beau que convaincant. Bien écrit, irrigué d'un usage large et inventif de la littérature, théoriquement incisif et parcimonieux, il renouvelle en profondeur notre connaissance des pratiques musicales mongoles. Il contribue aussi, et peut-être surtout, à la compréhension des manières dont les Mongols, et les humains d'une manière générale, s'attachent à certains sons qui en retour les attachent à d'autres choses.

\section{AUTEURS}

GRÉGORY DELAPLACE

g.delaplace@yahoo.fr 